\title{
Strain Mapping Using EBSD Cross Correlation and Raman Methods
}

\author{
M.D. Vaudin ${ }^{1}$, AJ Gayle ${ }^{1}$, L.H. Friedman ${ }^{1}$ and R.F. Cook ${ }^{1}$ \\ ${ }^{1 .}$ Materials Measurement Science Division, National Institute of Standards and Technology (NIST), \\ Gaithersburg, MD.
}

Diffraction measurement of strain has acquired a new tool in the past twenty years - cross-correlation electron backscatter diffraction (EBSD) [1], as championed by Wilkinson, Britton and many others. To assess the accuracy of the method, several types of sample have been used: e.g. $\mathrm{Si}_{1-x} \mathrm{Ge}_{x}$ deposited epitaxially on Si produces a calculable tetragonal strain state in the SiGe which has been studied to design an EBSD strain standard by Vaudin et al [2]; and indentations in Si produced by both wedge indenters [3], which create primarily a one-dimensional (1-D) strain field, and Vickers indenters [4] which create a twodimensional (2-D) strain field. We report EBSD and Raman strain measurements of the 2-D strain field around an indentation in single crystal Si produced by a conospherical indenter and discuss the good agreement between the two techniques; both techniques are potentially useful in enhancing the reliability of microelectromechanical systems (MEMS).

The test material for the indentation study was a $3 \mathrm{~mm}$ thick single crystal (001) Si disc, $35 \mathrm{~mm}$ in diameter. A square $3 \times 3$ array of test indentations was formed using a conospherical diamond probe of nominal $5 \mu \mathrm{m}$ tip radius. The peak load for the test indentations was $200 \mathrm{mN}$ and they were separated by $1 \mathrm{~mm}$ to avoid overlap of the indentation deformation fields. Two techniques for measuring strain maps that have appropriate spatial and strain resolutions for MEMS reliability applications, confocal Raman microscopy and electron backscatter diffraction, were used to map the strain fields around the indentation. The EBSD cross-correlation technique analyzes the distortion of the EBSD patterns (EBSPs) recorded from the sample relative to a reference EBSP recorded at the edge of the mapped area where the strain is assumed to be zero. The EBSPs were recorded using an Oxford-HKL system (Oxford Instruments, Abingdon, United Kingdom) installed on a JEOL 7100 SEM (JEOL USA Inc., Peabody, MA); the SEM was operated at a $20 \mathrm{keV}$ beam energy, a $2.3 \mathrm{nA}$ probe current and an electron spot size of $\approx 2 \mathrm{~nm}$. CrossCourt software version 3.2 [5] (CC3) was used to perform the analysis in which the pattern distortion at each point in the map was determined by cross-correlating the same regions of interest (ROIs) in the reference and strained pattern. In this study, 49 ROIs were used, yielding the pattern shifts at 49 points in each EBSP obtained at a mapped grid of sample positions. The initial step in the shift analysis was to remove the pattern shifts induced by the motion of the beam, and two methods were employed. In the first method, implemented by CC3, the shifts were calculated from the geometry of the mapping scan - the dimensions of the mapped area, step size and sample tilt. The results of this method have been published [6] and provided the results given below. The reference describes how Raman data were collected using a custom confocal Raman microscope; the EBSD results were used to predict the Raman shifts at each point in the scan. The Raman work is described in more detail in [3] In the second method of shift correction, the sample is translated parallel to the stage rotation axis until an undeformed (strain-free) region of the sample is under the beam and a scan is collected with the same map geometry. The shifts from this scan are subtracted from the strained scan shifts and the analysis proceeds as before. The effectiveness of this strategy will be discussed. In a related study, patterns have been collected from strainfree silicon mounted on a $45^{\circ}$ pre-tilted holder, tilted $25^{\circ}$ so the sample surface is at $70^{\circ}$, and then rotated by the stage through various angles to create misoriented sample surfaces. The shifts measured during a rectangular scan are determined by $\mathrm{CC} 3$ and correlated with the Euler angles determined at each point. 
References:

[1] AJ Wilkinson, G Meaden, and DJ Dingley, Mater. Sci. Technol. 22 (2006), p. 1271.

[2] MD Vaudin et al, Ultramicroscopy 148 (2015), p.94.

[3] LH Friedman et al, Ultramicroscopy 163 (2016), p. 75.

[4] TB Britton et al, Ultramicroscopy 135 (2013), p. 136.

[5] No endorsement by NIST is intended or implied by the identification of certain commercial equipment, instruments, or materials in this paper

[6] AJ Gayle et al, J Appl. Phys. 122 (2017), p. 205101.

Figure 1 shows color-filled contour maps of several of the strain components as determined by EBSD measurements in the strain field surrounding the indentation - note the different length scale in 1(c). As described in [6], these strain measurements were used to calculate the Raman shifts which allows the two measurement techniques to be compared in Figure 2 where the good agreement between EBSD-predicted and Raman-measured strains is shown.
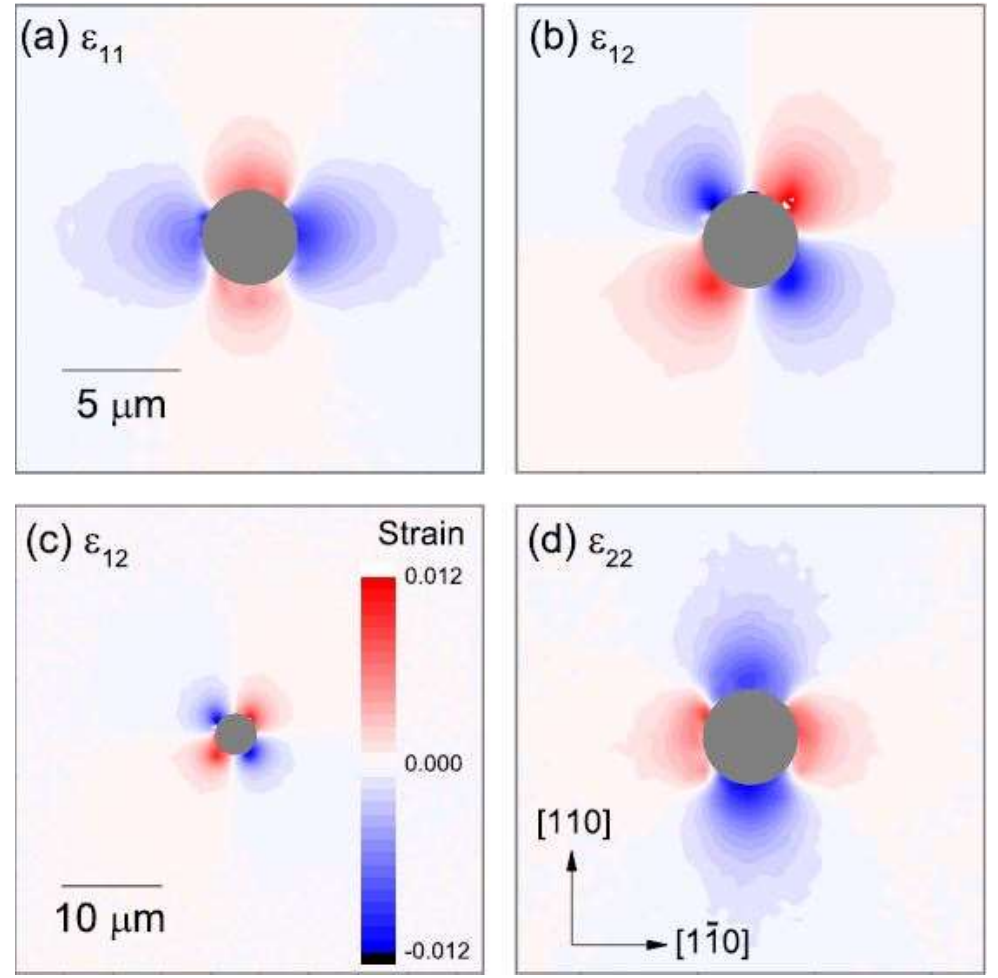

Figure 1. EBSD-measured strains: (a) $\varepsilon_{11}$, (b) and (c) $\varepsilon_{12}$, and (d) $\varepsilon_{22}$; gray circle marks position of residual indentation impression; note different length scale of $1(\mathrm{c})$; strain scale for all strains is in $1(\mathrm{c})$

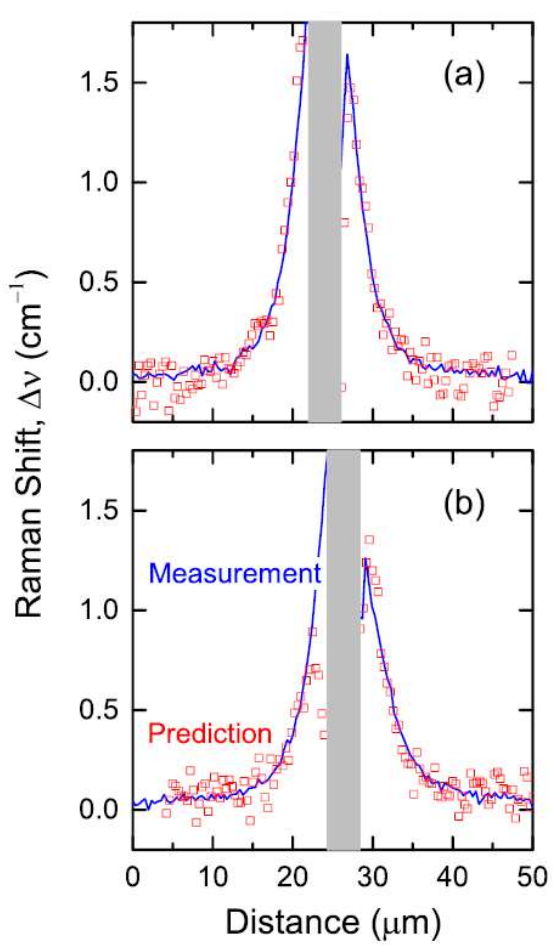

Figure 2. Line scans comparing measured Raman shifts to EBSDpredicted Raman shifts along cuts through the residual indentation impression in Fig. 1: $\quad$ 2(a) along [110]; 2(b) along [1 $1 \overline{1} 0]$ 